\title{
Bone mineral density and content are differentially impacted by aerobic and resistance training in the colon- 26 mouse model of cancer cachexia
}

Andy V. Khamoui ${ }^{1,2}$, Ming-Chia Yeh', Do-Houn Kim, Bong-Sup Park', Marcus L. Elam, ${ }^{1,3}$ Edward Jo 1,4, Bahram H. Arjmandi ${ }^{1,5}$ and Jeong-Su Kim ${ }^{1,5^{*}}$

\begin{abstract}
Background: Cancer cachexia is a debilitating paraneoplastic syndrome featuring unintended weight loss and skeletal muscle atrophy. Evidence suggests that bone health may also be compromised, further limiting mobility and quality of life. Aerobic and resistance training was recently reported to differentially affect skeletal muscle adaptations in cancer cachectic mice. The purpose of this investigation was to assess the effects of aerobic and resistance training on bone mineral density (BMD) and bone mineral content (BMC) in mice with colon-26 (C26) tumor-induced cachexia.

Methods: Twelve-month old Balb/c mice were aerobic-trained (wheel running 5 days/week) or resistance-trained (weighted ladder climbing 3days/week) for 8 weeks prior to C26 cell injection, followed by an additional three weeks of exercise. BMD and BMC were assessed pre- and post-training by dual-energy $x$-ray absorptiometry.

Results: Resistance-trained C26 mice lost total BMD by 7\% ( $p=0.06$ ), which did not occur in aerobic-trained C26 mice. In terms of pelvic bone, both resistance- and aerobic-trained C26 mice had significantly lower BMD values $(-12 \%, p=0.01$ and $-6 \%, p=0.04$, respectively), albeit to a lesser degree in aerobic-trained C26 mice. Furthermore, resistance-trained C26 mice tended to lose total BMC (-12\%), whereas aerobic-trained C26 mice maintained total BMC. In mice without C26 tumors, resistance training significantly increased total BMD $(+13 \%, p=0.001)$.

Conclusions: Aerobic and resistance training may differentially affect bone status in C26 cancer cachexia, with high resistance loading possibly being detrimental to total and pelvis BMD, a region expected to bear significant loading stress and contribute substantially to overall mobility. Because resistance training improved BMD in tumor-free mice, the C26 tumor burden appeared to impair the beneficial effect of resistance training on bone mass.
\end{abstract}

Keywords: Cachectic, C26 tumor, Exercise training, Body composition, DXA

\section{Background}

Approximately half of all cancer patients undergo cachexia, a paraneoplastic syndrome featuring unintended weight loss and skeletal muscle atrophy [1]. Tumorinduced cachexia severely impacts patient trajectory by impairing responsiveness to anti-cancer treatment and

\footnotetext{
* Correspondence: jkim6@fsu.edu

'Department of Nutrition, Food and Exercise Sciences, Florida State University, 432 Sandels Building, 120 Convocation Way, Tallahassee, FL 32306, USA

${ }^{5}$ Center for Advancing Exercise and Nutrition Research on Aging, Florida State University, Tallahassee, FL 32306, USA

Full list of author information is available at the end of the article
}

reducing quality and quantity of life [2]. Despite accounting for an estimated $20 \%$ of cancer-related deaths [3], cachexia remains an underappreciated issue in oncology and a source of frustration for patients and family members alike given the limited treatment options [1,4]. To address this unmet need, research has attempted to discern the principal causes and consequences of cachexia. Traditional emphasis has been placed on events specific to skeletal muscle mass and function; however, current frameworks describe a systemic condition in which multiple organs are reprogrammed or remodeled to generate the cachectic phenotype [5-7]. 
Although it is well known that skeletal muscle works in tandem with structures of the skeletal system to produce movement, and that both can be impacted concurrently by disease, the skeletal system has received less attention in cancer cachexia [8]. Given that several signaling events are common to both muscle and bone, inter-organ cross-talk and concurrent degeneration of bone in cancer cachexia is not surprising [8-10]. It was recently reported that metastasized bone contributes to cachexia-associated skeletal muscle dysfunction [11], suggesting regulation of skeletal muscle by bone. Furthermore, targeted treatment of cachexia via antibodies improved both lean body mass and bone mineral density [12]. These findings are strongly supportive of the close relationship between cachexia and bone health. Loss of structural integrity in bone substantially increases fracture risk, and ensuing fractures of load-bearing structures would seriously compromise mobility, quality of life, and prognosis. Therefore, bone health merits careful consideration and surveillance in patients that experience cachexia.

To address the numerous defects of cancer cachexia, a multi-modal therapeutic approach consisting of pharmaceutical agents, nutritional support, and programmed exercise training has been proposed $[2,13]$. In an effort to improve our understanding of therapeutic exercise applications, we recently investigated the impact of aerobic and resistance training on skeletal muscle plasticity in the colon-26 (C26) mouse model of cancer cachexia [14]. Although resistance training is known to stimulate muscle hypertrophy, we did not find overwhelming evidence of protection by resistance training against cancer-induced muscle atrophy. The marginal influence of resistance training in rodent cancer cachexia was also recently reported by another group of investigators [15]. In contrast, aerobic training has been shown to confer several benefits including maintenance of physical function and reduced hepatosplenomegaly [14]. Furthermore, others reported aerobic exercise to not only inhibit muscle atrophy, but also prolong the survival time of $\mathrm{C} 26$ mice [16]. These results suggest differential adaptations to the classical modes of exercise training in rodent models of cancer cachexia. Although exercise training is known to regulate bone remodeling [17], the influence of different exercise modalities on bone status in cancer cachexia is not well-defined.

The purpose of the present study was to assess the extent to which aerobic and resistance training impacts bone mineral density (BMD) and bone mineral content (BMC) in the colon-26 (C26) mouse model of cancer cachexia. We were particularly interested in whether the divergent responses of $\mathrm{C} 26$ mice to aerobic and resistance training might also be reflected in bone.

\section{Methods}

\section{Mice and experimental design}

Twelve-month old Balb/c female mice (Harlan Laboratories Inc., USA) were randomly assigned to one of three groups: Control $(n=17)$, Resistance Training (RT, $n=16$ ), or Aerobic Training (AT, $n=16$ ). Mice assigned to $\mathrm{RT}$ or AT were given a 1-week period to familiarize with the exercises. All mice then underwent preexperiment assessments of physical function and body composition. Next, mice in RT and AT performed their respective exercises for 8 weeks. Half of the mice in each initial group were then injected s.c. with colon-26 (C26) tumor cells, followed by 3 additional weeks of training. Physical function and body composition measurements were repeated after training, and tissues were collected $48 \mathrm{~h}$ after euthanasia for in vitro analysis. In all, six groups were investigated including Control $(n=8), \mathrm{C} 26(n=9)$, AT $(n=8), \mathrm{AT}+\mathrm{C} 26(n=8)$, RT $(n=8)$, and RT + C26 $(n=8)$. During the experiment, two RT $+\mathrm{C} 26$ mice met the humane endpoints and were euthanized accordingly. The remaining $\mathrm{RT}+\mathrm{C} 26$ mice $(n=6)$ completed the study. All mice were housed individually and fed standard chow and water ad libitum and maintained on a 12:12 h light:dark cycle. Study approval was obtained by the Institutional Animal Care and Use Committee at Florida State University before initiating the project. Physical function measurements, select body composition parameters (total body, lean, and fat mass), and in vitro skeletal muscle data have been previously published [14]. All data pertaining to bone are reported in the present study.

\section{Aerobic and resistance training}

Resistance exercise was conducted at a frequency of 3 days per week using weighted ladder climbing [18]. Overload was applied by attaching a padded foam clip with weight at the base of the tail. To increase the loading, washers or paper clips were added to the foam clip. The initial resistance attached was $50 \%$ of body weight followed by $10 \%$ increases bi-weekly. The specific loading schedule for weeks $1-2,3-4,5-6,7-8$, and 9-11 were as follows: RT) $14 \pm 1,17 \pm 1,20 \pm 1,23 \pm 1$, and $26 \pm 1 \mathrm{~g}$; and RT + C26) $14 \pm 1,17 \pm 1,19 \pm 1,22 \pm 1$, and $25 \pm 1 \mathrm{~g}$. The daily protocol began with a single repetition without weight as a warm-up, followed by 5 sets of 3 repetitions at the prescribed loads. One and $2 \mathrm{~min}$ of rest were provided between repetitions and sets, respectively. Aerobic exercise was performed at a frequency of 5 days per week, for $60 \mathrm{~min}$ per day using motorized wheels (Model 80800A, Lafayette Instrument, Lafayette, IN). Before beginning the main exercise session, mice performed a 15-min warm-up that consisted of speeds increased progressively to the intensity to be used during the main session. The main session speeds for all aerobic-trained 
groups during weeks $1-2,3-5,6-7$, and $8-11$ of training were $5.0,6.0,7.0$, and $6.5 \mathrm{~m} / \mathrm{min}$, respectively. The speed was reduced to a fast walk $(6.5 \mathrm{~m} / \mathrm{min})$ during the later stages of the project because the mice exhibited a tendency to grip the bars of the motorized wheels and rotate in circles instead of exercising at the prescribed speed of $7.0 \mathrm{~m} / \mathrm{min}$. This speed reduction was sufficient to increase compliance and allow the mice to perform continuous exercise with minimal bar grasping.

\section{C26 tumor cell injection}

C26 tumor-bearing mice are widely used to study cancer cachexia [19-21]. The C26 cells were cultured in a humidified 5\% $\mathrm{CO}_{2}$ incubator using RPMI 1640 supplemented with $1 \%$ penicillin/streptomycin ( $\mathrm{vol} / \mathrm{vol}$ ) and $10 \% \mathrm{FBS}$ (vol/vol). At sub-confluency, cells were trypsinized for harvesting $(0.05 \%$, Gibco) and pelleted by centrifugation. The supernatant was then removed and the pellet resuspended in PBS. Viable cells were counted by trypan blue staining. Mice in C26 groups were gently restrained and injected s.c. in the upper back with a $0.1 \mathrm{ml}$ cell suspension containing $5.0 \times 10^{5}$ cells. Mice assigned to tumor-free groups were injected at the same site with $0.1 \mathrm{ml}$ sterile PBS [20, 22].

\section{Dual energy $x$-ray absorptiometry (DXA)}

Whole body BMD and BMC as well as regional BMD of the arms, legs, and pelvis were determined by DXA with software provided by the manufacturer (iDXA Lunar, GE). For the pre-experiment measurement, the mice were first anesthetized i.p. with ketamine/xylazine $(70 / 3 \mathrm{mg} / \mathrm{kg}$ body wt) and positioned prone on the scanner bed. Each full length scan lasted approximately $1 \mathrm{~min}$. Postexperiment measurements were taken after sacrifice by ketamine/xylazine overdose. The tumors were removed prior to the final scan in accordance with previous work [23, 24]. The region of interest (ROI) for the arms, legs, and pelvis were defined as the junction of the glenoid fossa and humeral head to the distal phalange, the proximal knee to the distal toe, and the superior to the inferior aspect of the pelvis, respectively. A sample scan is shown in Fig. 1.

\section{Statistical analysis}

All data have been reported as mean \pm SE. To evaluate effects of the C26 tumor on BMD and BMC, a pre-planned 2-way ANOVA was conducted using the factors of group (Control, C26) and time (pre-, post-experiment). To evaluate effects of exercise training in C26 mice, 2-way ANOVA (group $x$ time) was used to evaluate pre- to posttraining responses in $\mathrm{C} 26$, aerobic-trained $\mathrm{C} 26$ ( $\mathrm{AT}+$ $\mathrm{C} 26)$, and resistance-trained $\mathrm{C} 26$ mice $(\mathrm{RT}+\mathrm{C} 26)$. Initial analyses revealed compromised bone parameters in the resistance-trained C26 mice; therefore, to discern if resistance training alone could contribute to the observed degeneration, follow-up 2-way ANOVAs (group x time) were then conducted to evaluate longitudinal responses in resistance-trained mice with and without the $\mathrm{C} 26$ tumor (i.e. RT and RT + C26). Significant interactions and main effects were followed up with Tukey's HSD or t-tests. Pvalues $\leq 0.05$ were considered significant.

\section{Results}

Loss of total BMC and pelvis BMD in C26-tumor induced cachexia

To first verify the induction of weight loss (i.e. cachexia), DXA-determined total body mass was analyzed by 2-way ANOVA (previously published in [14] and
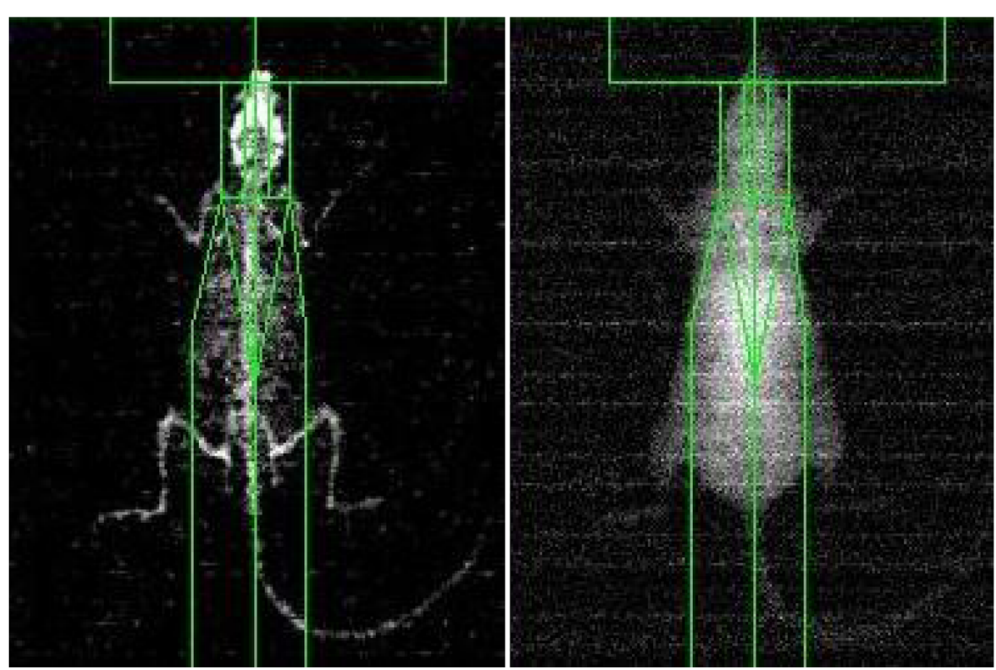

Fig. 1 Representative scan obtained from dual-energy x-ray absorptiometry 
reported here for descriptive purposes only). Preexperiment total body mass was not different between Control and C26 $(p>0.05)$. From pre- to post-experiment, body mass significantly decreased in C26 $(-8 \%, p<0.01)$ but was not altered in Control $(p>0.05)$, indicating that the C26 tumor load caused unintended weight loss. For total BMC and BMD, 2-way ANOVAs found no significant interaction of group $\mathrm{x}$ time $(p>0.05)$. However, there was a significant main effect of time $(p \leq 0.05)$ on total BMC in which follow-up tests revealed losses in both Control $(-9 \%, p=0.06)$ and C26 $(-14 \%, p=0.02)$ (Fig. 2a).
Additionally, there was a significant main effect of time on total BMD $(p \leq 0.05)$. Follow-up tests showed the effect to be driven by a significant increase in Control $(+4 \%, p=0.04)$ whereas there was no difference over time in C26 ( $p>0.05)$ (Fig. 2b). Analysis of regional bone mineral density found no significant interactions or main effects in the arms or legs $(p>0.05)$ (Fig. 2c). However, there was a significant interaction on pelvis $\operatorname{BMD}(p \leq 0.05)$. At pre-experiment, pelvis BMD tended toward greater values in $\mathrm{C} 26$ vs. Control $(+5 \%, p=0.06)$ (Fig. 2c). When examined longitudinally, pelvis BMD

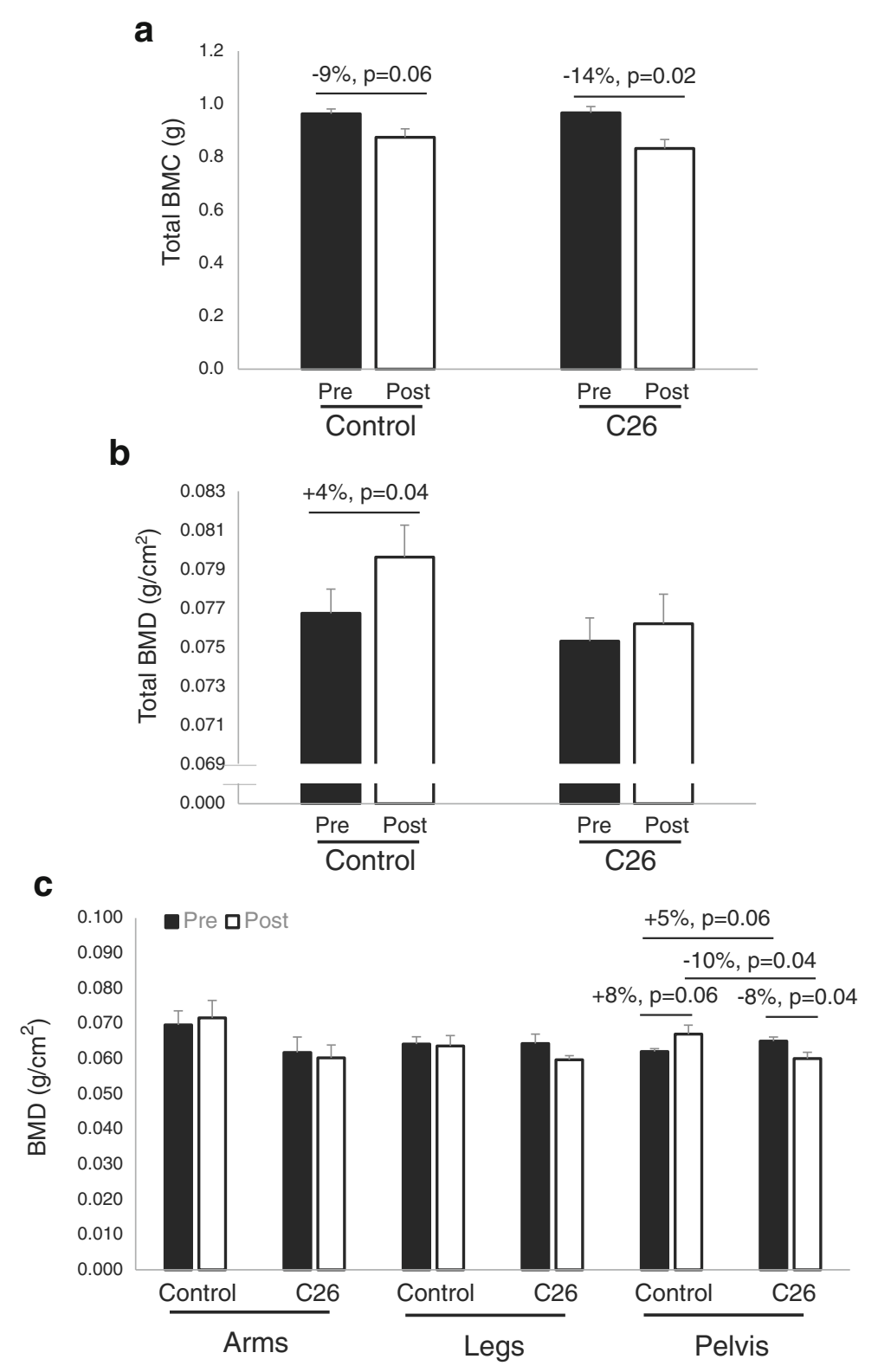

Fig. 2 Loss of total bone mineral content (BMC) and pelvis-specific bone mineral density (BMD) in colon-26 (C26) tumor-induced cachexia, Dual-energy $x$-ray absorptiometry was used to evaluate effects of the C26 tumor on total BMC (a), total BMD (b), and regional BMD (c). Values are mean $\pm \mathrm{SE}, n=8-9 /$ group 
tended to increase in Control $(+8, p=0.06)$ while C26 significantly decreased $(-8 \%, p=0.04)$ (Fig. $2 \mathrm{c}$ ). When compared at post-experiment, pelvis BMD was significantly lower in C26 $(-10 \%, p=0.04)$ (Fig. 2c).

Loss of total BMD and BMC in resistance- but not aerobictrained C26 mice

To explore the impact of aerobic and resistance training on bone health of $\mathrm{C} 26$ tumor-bearing mice, twoway ANOVA was conducted using the factors of group $(\mathrm{C} 26$, $\mathrm{AT}+\mathrm{C} 26$, and $\mathrm{RT}+\mathrm{C} 26)$ and time (preand post-training). ANOVA found a significant main effect of time on total BMC $(p \leq 0.05)$. Post hoc tests showed the effect be driven by a significant decline in C26 $(-14 \%, p=0.02)$ and a tendency toward loss in $\mathrm{RT}+\mathrm{C} 26(-12 \%, p=0.11) \quad$ (Fig. 3a). Total BMC in AT + C26 did not change over time $(p>0.05)$ (Fig. 3a). For total BMD, there was a significant interaction of group $\mathrm{x}$ time $(p \leq 0.05)$. Follow-up tests indicated no differences between the three groups at pre-training $(p>0.05)$ (Fig. 3b). When evaluated longitudinally, $\mathrm{RT}+\mathrm{C} 26$ tended to lose total BMD $(-7 \%, p=0.06)$ whereas $\mathrm{C} 26$ and $\mathrm{AT}+\mathrm{C} 26$ were not significantly altered $(p>0.05)$ (Fig. 3b). When compared at posttraining, total BMD was significantly lower in RT + C26 vs. C26 $(-8 \%, p=0.05)$ (Fig. 3b). Total BMD in $\mathrm{RT}+\mathrm{C} 26$ also tended to be lower than $\mathrm{AT}+\mathrm{C} 26$ at post-training $(-8 \%, p=0.07)$ (Fig. $3 \mathrm{~b})$. In the regional assessments, ANOVA did not detect significant differences at the arms or legs $(p>0.05)$ (Figs. 4a and b). At the pelvis however, there was a significant main effect of time that follow-up tests showed to be driven by pre- to post-training reductions in all three C26 groups ( $-6-12 \%, p=0.01-0.04$ ) (Fig. 4c). Longitudinal changes in the total body mass of exercisetrained C26 mice were previously reported [14] and

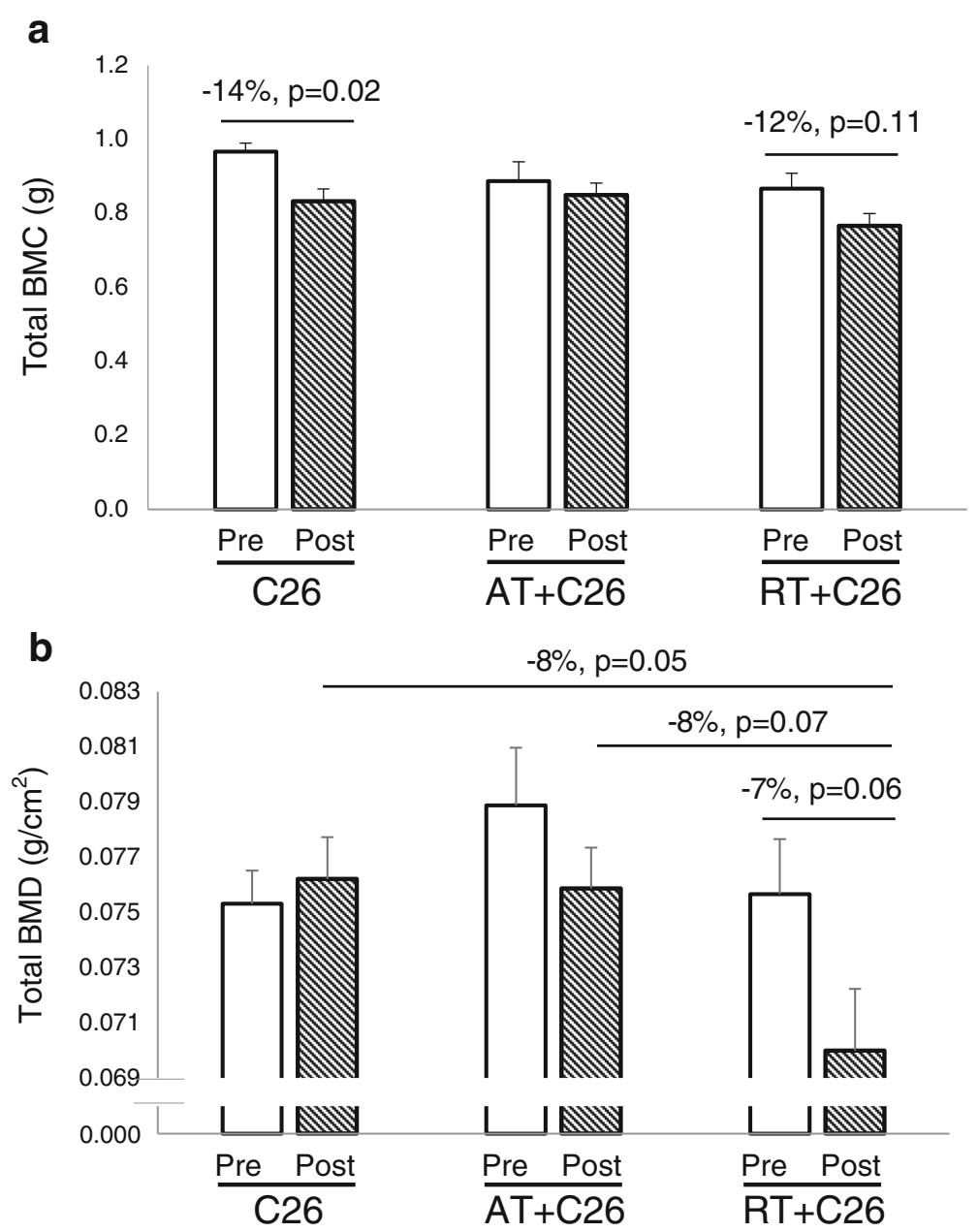

Fig. 3 Differential response of total bone mineral content (BMC) and bone mineral density (BMD) to aerobic and resistance training in colon-26 (C26) tumor-induced cachexia. Dual-energy x-ray absorptiometry was used to assess the effect of each training mode on total BMC (a) and total BMD (b) in mice bearing the $\mathrm{C} 26$ tumor. Values are mean $\pm \mathrm{SE}, n=6-9 /$ group. $A T+C 26$ aerobic-trained C26 mice. $R T+C 26$ resistance-trained C26 mice 


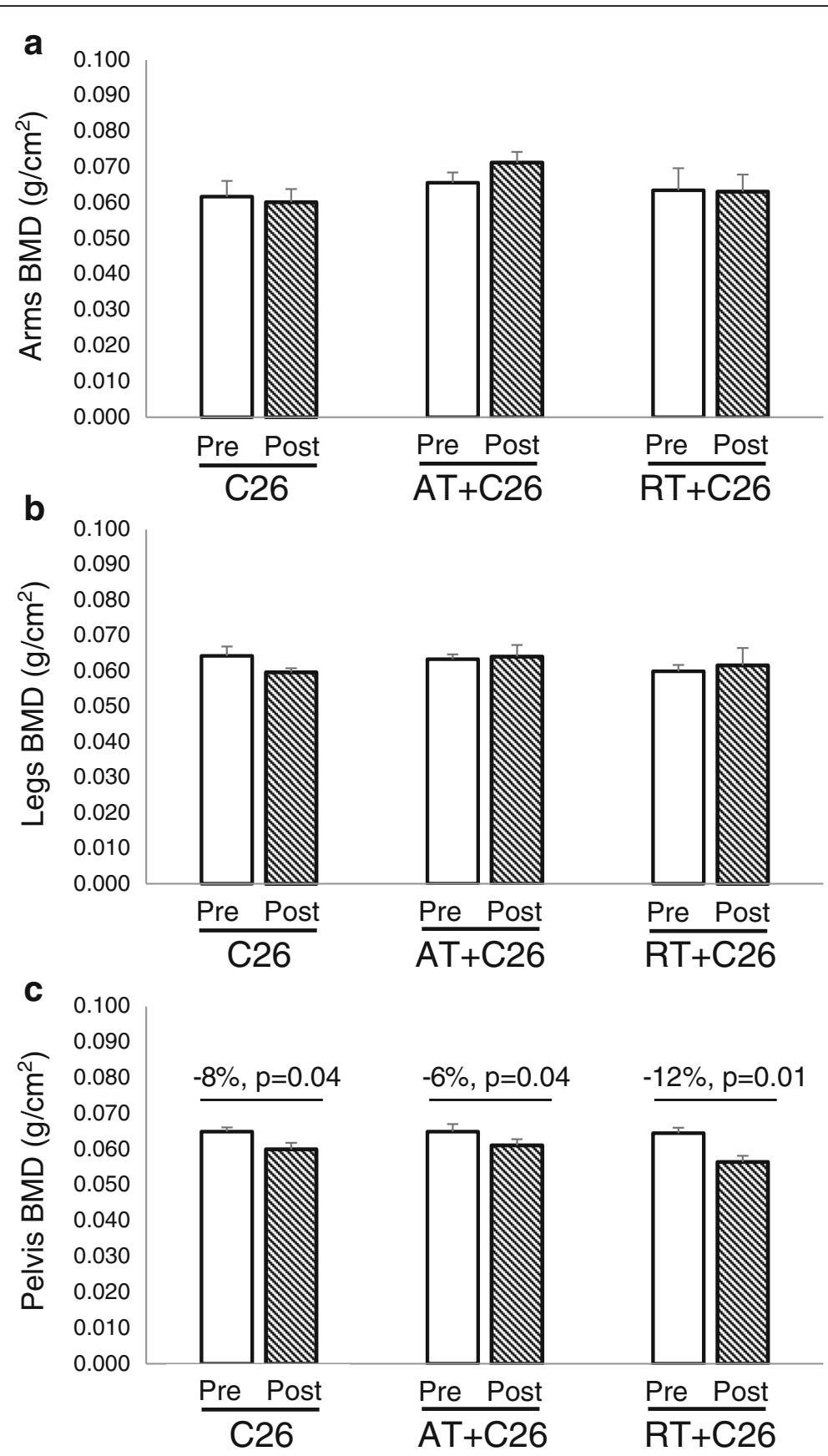

Fig. 4 Regional bone mineral density (BMD) responses to aerobic and resistance training in colon-26 (C26) tumor-induced cachexia. Dual-energy x-ray absorptiometry was used to determine the effect of each training mode on BMD of the arms (a), legs (b), and pelvis (c) in mice bearing the C26 tumor. Values are mean $\pm \mathrm{SE}, n=6-9 /$ group. $A T+$ C26 aerobic-trained C26 mice. $R T+$ C26 resistance-trained C26 mice

are provided here for descriptive purposes. No pretraining differences were noted, however, total body mass significantly decreased over time $(p<0.01)$ in C26 (-8\%), AT + C26 (-18\%), and RT + C26 (-15\%). The three $\mathrm{C} 26$ groups were not significantly different when compared at post-training $(p>0.05)$.
Increased total BMD in tumor-free resistance-trained mice To explore whether the deficits in resistance-trained C26 mice related to the nature of the resistance training program itself, 2-way ANOVAs were conducted to evaluate longitudinal changes in Controls, resistance-trained mice without tumors (RT), and resistance-trained C26 
mice $(\mathrm{RT}+\mathrm{C} 26)$. There was a significant interaction of group $\mathrm{x}$ time on total $\mathrm{BMD}(p \leq 0.05)$. Pre-experiment total BMD was not different between the three groups $(p>0.05)$ (Fig. 5a). From pre- to post-experiment, total BMD significantly increased in Control $(+4 \%, p=0.04)$ and RT $(+13 \%, p=0.001)$, whereas $\mathrm{RT}+\mathrm{C} 26$ demonstrated a deficit $(-7 \%, p=0.06)$ (Fig. 5a). When compared at postexperiment, total BMD was greater in RT vs. Control $(+8 \%$, $p=0.06)$ and $\mathrm{RT}+\mathrm{C} 26(+23 \%, p<0.001)$ (Fig. 5a). There was also a significant interaction of group $\mathrm{x}$ time on pelvis $\operatorname{BMD}(p \leq 0.05)$. Pre-experiment values were not different between the three groups $(p>0.05)$ (Fig. 5b). From pre- to post-experiment, pelvis BMD increased in Control $(+8 \%, p=0.06)$ and RT $(+7 \%, p=0.07)$, but decreased in $\mathrm{RT}+\mathrm{C} 26(-12 \%, p=0.01)$ (Fig. 5b). At post-experiment, pelvis BMD in RT was greater than $\mathrm{RT}+\mathrm{C} 26(+25 \%, p=0.001)$, but not different from Control $(p>0.05)$ (Fig. 5b). Control also had greater pelvis BMD compared to $\mathrm{RT}+\mathrm{C} 26(+19 \%, p=0.009)$
(Fig. 5b). There were no significant interactions or main effects for BMD at the arms and legs $(p>0.05)$ (data not shown).

\section{Discussion}

This investigation evaluated the impact of aerobic and resistance training on bone status in C26 tumor-bearing mice. Our principal findings were that resistance-trained C26 mice lost total BMD $(-7 \%, p=0.06)$, which did not occur in aerobic-trained C26 mice $(p>0.05)$ (Fig. 3b). Resistance-trained C26 mice also showed a significant decrease in pelvis BMD (Fig. 4c). Although aerobic-trained C26 also showed a significant reduction of pelvis BMD, the degree of loss displayed by resistance-trained C26 mice was approximately twice as great (Fig. 4c). In addition, resistance-trained $\mathrm{C} 26$ mice tended to lose total bone mineral content $(-12 \%, p=0.11)$ whereas aerobictrained C26 mice maintained total bone mineral content $(p>0.05)$ (Fig. 3a). These findings suggest that aerobic

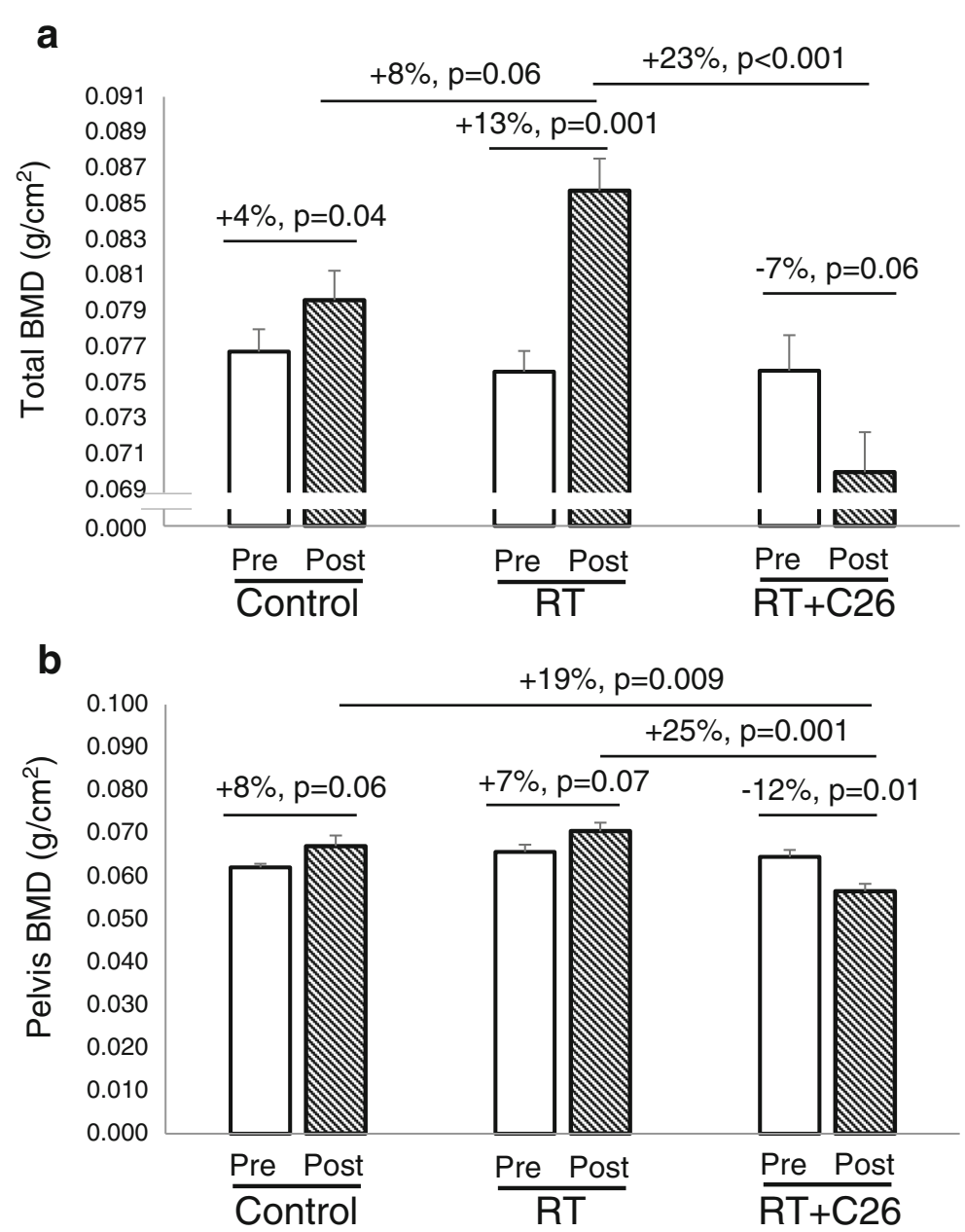

Fig. 5 Colon-26 (C26) tumor-induced cachexia impaired the beneficial effect of resistance training on total bone mineral density (BMD). Dual-energy x-ray absorptiometry was used to evaluate the impact of resistance training in mice with and without C26 tumors on total BMD (a) and pelvis-specific BMD (b). Values are mean $\pm \mathrm{SE}, n=6-8$ /group. $R T$ resistance-trained mice without tumors. $R T+C 26$ resistance-trained C26 mice 
and resistance training may differentially affect BMD and BMC in cancer cachexia, with high resistance loading possibly being detrimental to bone health.

\section{Changes in BMD and BMC in colon-26 cancer cachexia}

In this murine model of cancer cachexia, C26 mice experienced a $14 \%$ decrease in total BMC from pre- to post-experiment (Fig. 2a). During the same period, tumor-free Control mice experienced a 9\% loss of total BMC that tended toward significance (Fig. 2a). Because we used mice that were 12 months of age at the study's commencement, these findings suggest that age may have contributed to some of the mineral content loss. Nonetheless, C26 mice exhibited an additional 5\% decrease that presumably occurred as a result of the tumor. Interestingly, total BMD in Control significantly increased by $4 \%$ from pre- to post-experiment whereas C26 mice did not show any significant change (Fig. 2b). Since BMD accounts for BMC relative to the area evaluated, the loss of total BMC but unchanged BMD in C26 mice (Figs. 2a and b) suggests remodeling that possibly expanded bone area in order to maintain total density. In contrast, mice with Lewis lung cancer cachexia showed a statistically significant $5 \%$ loss in total bone mineral density [8], which may relate to differences in the model used and age of the mice. When analyzed at specific regions, C26 mice showed a significant decrease of BMD at the pelvis but not the arms or legs (Fig. 2c). Given the load bearing role of the pelvis, mobility would likely be compromised and quality of life reduced. It may also be worth mentioning that myofiber cross-sectional area of the gastrocnemius (reported previously in [14]) correlated significantly with delta total BMD $\left(r=0.47, R^{2}=0.22, p<0.01\right)$ and delta pelvis BMD $\left(r=0.58, R^{2}=0.33, p<0.01\right)$ (data not shown). This suggests that changes in BMD parallel changes in muscle mass in C26 cancer cachexia. Such alterations in bone add to the existing number of abnormalities that may require surveillance during cancer cachexia.

Our use of DXA to assess skeletal properties in the present work merits further discussion, particularly with respect to the technical aspects of this device. Although widely used in clinical and research settings, DXA measures the average bone mineral content in a 2dimensional area, and is unable to assess changes in bone microstructure and tissue density [25]. In contrast, 3-dimensional imaging systems such as quantitative computed tomography can distinguish these parameters in both cortical and cancellous bone [25]. This is particularly important since microarchitecture is an indicator of bone quality [26]. Therefore, future work in this area should incorporate assessments of bone microstructure to better understand changes that occur during cancer cachexia, as well as the efficacy of exercise therapies.

\section{Differential response of BMD and BMC to aerobic and resistance training in C26 mice}

We previously reported divergent effects of aerobic and resistance training on skeletal muscle adaptations in the same mouse model of cancer cachexia [14]. Specifically, we did not find compelling evidence of a favorable countermeasure effect by resistance training. Our current findings further support a differential response to aerobic and resistance training in cancer cachexia, with specific reference to bone status. Resistance-trained C26 mice displayed a $12 \%$ drop in total BMC that although not statistically significant ( $p=0.11$ ) (Fig. 3a), was comparable to the statistically significant deficit experienced by their sedentary C26 counterparts $(-14 \%, p=0.02)$ (Fig. 2a). In contrast, aerobic-trained $\mathrm{C} 26$ mice maintained total BMC during the same period (Fig. 3a). Furthermore, resistance-trained C26 mice demonstrated loss of total BMD that nearly reached statistical significance $(-7 \%, p=0.06)$ (Fig. 3b), whereas aerobic-trained C26 mice did not experience significant changes (Fig. 3b). With respect to pelvis BMD, exercise training was unable to prevent deficits over time as all three $\mathrm{C} 26$ groups showed significant pre- to post-training reductions (Fig. 4c). It may be worth noting that the degree of pelvic $\mathrm{BMD}$ loss in resistance-trained $\mathrm{C} 26$ mice was twice as much as their aerobic-trained counterparts (Fig. 4c). Therefore, it could be suggested that resistance training may have exacerbated deterioration of pelvic BMD during cancer cachexia.

\section{Resistance training enhanced total BMD in tumor-free mice}

Based on the adverse impact of high-resistance loading in tumor-bearing mice, we then conducted additional analysis on the longitudinal bone responses in Control, tumor-free resistance-trained mice (RT), and resistancetrained C26 mice $(\mathrm{RT}+\mathrm{C} 26)$. The purpose of this follow-up was to define and compare the bone responses to resistance training in mice with and without C26 tumor-induced cachexia. Such an approach was important to discern whether the resistance training program alone may have contributed to losses of BMD seen in $\mathrm{RT}+\mathrm{C} 26$ (Fig. 3b), or if the degeneration resulted from the combined effect of resistance training and tumor load. We found that tumor-free resistance-trained mice significantly increased total BMD by $13 \%$ from preto post-training, with values at post-training being greater than Control $(+8 \%, p=0.06)$ and $\mathrm{RT}+\mathrm{C} 26$ $(+23 \%, p<0.001)$ (Fig. 5a). Tumor-free resistancetrained mice also tended to increase BMD of the pelvis $(+7 \%, p=0.07)$, with values at post-training being greater than $\mathrm{RT}+\mathrm{C} 26(+25 \%, p=0.001)$ (Fig. 5b). These improvements in BMD after resistance training are consistent with the general assertion that exercise-induced 
mechanical loading benefits bone health [27]. Therefore, our data suggests the total BMD loss in resistance-trained C26 mice to result from the combined stress of mechanical overload and tumor burden rather than their individual impact. This idea is supported by the minimal effect of the C26 tumor alone on total BMD (Fig. 2b), and the improvement in total BMD of resistance-trained mice without tumors (Fig. 5a).

The possibility exists that metastatic bone disease could have predisposed the resistance-trained C26 mice to skeletal complications. Although it is well-established that colorectal cancer does not frequently metastasize to bone [28], mice bearing the C26 tumor have been previously used to study bone metastasis [29], and possible pathological fracture is a significant concern [30]. However, in prostate cancer patients with bone metastasis, 12-weeks of resistance training was well-tolerated, did not cause skeletal complications, and significantly improved physical function and body composition [31]. Although it would be difficult to make direct comparisons with this study given the different cancer type and model (patients vs. mice), resistance training appeared to be beneficial even in the presence of metastatic disease. Therefore, the deficits observed in the present study may only be applicable to resistance loading in the C26 model of cancer cachexia.

Lastly, although the impact of aerobic training in tumor-free mice was not a principal aim of this study, it may be worth discussing how this other major exercise modality impacted bone. We performed a secondary analysis on longitudinal changes in total BMD in tumorfree aerobic-trained mice (AT) and aerobic-trained C26 mice $(\mathrm{AT}+\mathrm{C} 26)$ by 2 -way ANOVA. There was a significant interaction of group by time on total BMD $(p \leq 0.05)$ (data not shown). At pre-training, no differences were noted between the groups $(p>0.05)$. From pre- to posttraining, total BMD significantly increased in tumor-free aerobic-trained mice $(+7 \%, p=0.025)$, whereas aerobictrained C26 mice showed no significant change $(p>0.05)$. These findings suggests that in our hands, aerobic training can also promote increased bone mass in tumor-free mice, but to a lesser extent than resistance training $(+7 \%$ in AT vs. $+13 \%$ in $\mathrm{RT}$ ). It also indicates that despite a reduction in training speed toward the end of the study to promote increased compliance, a positive adaptive response was still evident.

\section{Perspectives and Conclusions}

Therapies for cancer cachexia are likely to consist of multiple arms such as pharmaceutical agents, nutritional support, and exercise training. Because skeletal muscle atrophy and dysfunction are hallmark features of this paraneoplastic syndrome, skeletal muscle is a principal target in cancer cachexia therapies. Bone health is an underappreciated aspect of cancer cachexia despite being in close proximity to skeletal muscle, acting coordinately to generate movement, and cross-talk mechanisms regulating both organs. Aerobic and resistance training, the classical exercise modalities often used in clinical and sport settings, have been reported to induce different skeletal muscle adaptations in rodent models of cancer cachexia. Resistance training in particular did not appear to provide convincing protective effects. In agreement, we found BMD and BMC to also be differentially affected by aerobic and resistance training. Specifically, application of high resistance loading in the presence of a cachexia-promoting tumor may have facilitated the degeneration of bone mass. Additional work is needed to understand how resistance loading during C26 tumor burden may have affected regulators of bone metabolism (e.g. osteocalcin, ALP, TRAF). Follow-up investigations should also conduct histological analysis and 3-dimensional imaging by micro-CT scanning to determine microstructural characteristics and estimate biomechanical parameters. These important techniques were not performed in the present work and are limitations of this study. Incorporation of these approaches would provide better insight on skeletal changes during cancer cachexia, such as cortical bone characteristics, which is particularly relevant because a majority of non-vertebral fractures occur at cortical sites, age-related deterioration of appendicular bone is mostly cortical, and much of this bone loss arises from intracortical remodeling leading to porosity [32, 33]. Such technical approaches would also help to evaluate the efficacy of exercise therapies. Nevertheless, our findings illustrate the importance of applying disease-specific exercise therapies rather than general programming principles across different pathologies.

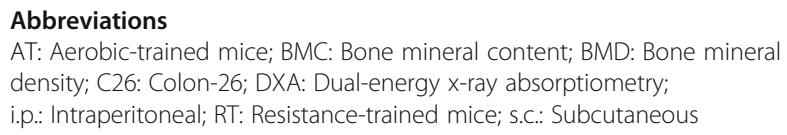

\section{Acknowledgements}

The authors extend their sincere thanks to Dr. Donna McCarthy (Marquette University) for kindly providing the C26 tumor cells.

\section{Funding \\ Study supported by Dissertation Research Grants from The Graduate School and College of Human Sciences at Florida State University.}

Availability of data and materials

All data generated or analyzed during this study are included in this published article.

\section{Authors' contributions}

Study design: AVK, BHA, JSK; Data collection and analysis: AVK, MCY, DHK, BSP, MLE, EJ, JSK; Drafted manuscript: AVK, JSK; Approved final version: AVK, MCY, DHK, BSP, MLE, EJ, BHA, JSK.

Competing interests

The authors declare that they have no competing interests. 


\section{Consent for publications}

Not applicable.

\section{Ethics approval}

All procedures were approved by the Institutional Animal Care and Use Committee at Florida State University (Protocol \#1242).

\section{Publisher's Note}

Springer Nature remains neutral with regard to jurisdictional claims in published maps and institutional affiliations.

\section{Author details}

${ }^{1}$ Department of Nutrition, Food and Exercise Sciences, Florida State University, 432 Sandels Building, 120 Convocation Way, Tallahassee, FL 32306, USA. ${ }^{2}$ Department of Exercise Science and Health Promotion, Florida Atlantic University, Boca Raton, FL 33431, USA. ${ }^{3}$ Department of Human Nutrition and Food Science, California State Polytechnic University, Pomona, Pomona, CA

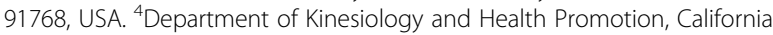
State Polytechnic University, Pomona, CA 91768, USA. ${ }^{5}$ Center for Advancing Exercise and Nutrition Research on Aging, Florida State University, Tallahassee, FL 32306, USA.

Received: 6 January 2017 Accepted: 5 May 2017

Published online: 08 June 2017

\section{References}

1. von Haehling S, Anker SD. Cachexia as a major underestimated and unmet medical need: facts and numbers. J Cachex Sarcopenia Muscle. 2010;1(1):1-5.

2. Fearon K, Arends J, Baracos V. Understanding the mechanisms and treatment options in cancer cachexia. Nat Rev Clin Oncol. 2013;10(2):90-9.

3. Inagaki J, Rodriguez V, Bodey GP. Proceedings: causes of death in cancer patients. Cancer. 1974;33(2):568-73.

4. Reid J, McKenna HP, Fitzsimons D, McCance TV. An exploration of the experience of cancer cachexia: what patients and their families want from healthcare professionals. Eur J Cancer Care (Engl). 2010;19(5):682-9.

5. Argiles JM, Busquets S, Stemmler B, Lopez-Soriano FJ. Cancer cachexia: understanding the molecular basis. Nat Rev Cancer. 2014;14(11):754-62.

6. Petruzzelli M, Wagner EF. Mechanisms of metabolic dysfunction in cancerassociated cachexia. Genes Dev. 2016;30(5):489-501.

7. Porporato PE. Understanding cachexia as a cancer metabolism syndrome. Oncogenesis. 2016:5:e200.

8. Choi E, Carruthers K, Zhang L, Thomas N, Battaglino RA, Morse LR, Widrick J. Concurrent muscle and bone deterioration in a murine model of cancer cachexia. Physiol Rep. 2013;1(6):e00144.

9. Kandarian S. The molecular basis of skeletal muscle atrophy-parallels with osteoporotic signaling. J Musculoskelet Neuronal Interact. 2008;8(4):340-1.

10. Waning DL, Guise TA. Cancer-associated muscle weakness: What's bone got to do with it? Bonekey Rep. 2015;4:691.

11. Waning DL, Mohammad KS, Reiken S, Xie W, Andersson DC, John S, Chiechi A, Wright LE, Umanskaya A, Niewolna M, et al. Excess TGF- $\beta$ mediates muscle weakness associated with bone metastases in mice. Nat Med. 2015;21(11):1262-71.

12. Greco SH, Tomkötter $L$, Vahle AK, Rokosh R, Avanzi A, Mahmood SK, Deutsch M, Alothman S, Alqunaibit D, Ochi A, et al. TGF- $\beta$ blockade reduces mortality and metabolic changes in a validated murine model of pancreatic cancer cachexia. PLoS One. 2015;10(7):e0132786.

13. Maddocks M, Hopkinson J, Conibear J, Reeves A, Shaw C, Fearon KC. Practical multimodal care for cancer cachexia. Curr Opin Support Palliat Care. 2016;10(4):298-305

14. Khamoui AV, Park BS, Kim DH, Yeh MC, Oh SL, Elam ML, Jo E, Arjmandi BH, Salazar G, Grant SC, et al. Aerobic and resistance training dependent skeletal muscle plasticity in the colon-26 murine model of cancer cachexia. Metabolism. 2016;65(5):685-98.

15. das Neves W, Alves CR, de Almeida NR, Guimaraes FL, Ramires PR, Brum PC, Lancha Jr AH. Loss of strength capacity is associated with mortality, but resistance exercise training promotes only modest effects during cachexia progression. Life Sci. 2016;163:11-22.

16. Pigna E, Berardi E, Aulino P, Rizzuto E, Zampieri S, Carraro U, Kern H, Merigliano S, Gruppo M, Mericskay M, et al. Aerobic exercise and pharmacological treatments counteract cachexia by modulating autophagy in colon cancer. Sci Rep. 2016;6:26991.
17. Martyn-St James M, Carroll S. Effects of different impact exercise modalities on bone mineral density in premenopausal women: a meta-analysis. J Bone Miner Metab. 2010;28(3):251-67.

18. Lee S, Barton ER, Sweeney HL, Farrar RP. Viral expression of insulin-like growth factor-I enhances muscle hypertrophy in resistance-trained rats. J Appl Physiol. 2004;96(3):1097-104.

19. Di Marco S, Cammas A, Lian XJ, Kovacs EN, Ma JF, Hall DT, Mazroui R, Richardson J, Pelletier J, Gallouzi IE. The translation inhibitor pateamine A prevents cachexia-induced muscle wasting in mice. Nat Commun. 2012;3:896.

20. Diffee GM, Kalfas K, Al-Majid S, McCarthy DO. Altered expression of skeletal muscle myosin isoforms in cancer cachexia. Am J Physiol Cell Physiol. 2002;283(5):C1376-1382

21. Zhou X, Wang JL, Lu J, Song Y, Kwak KS, Jiao Q, Rosenfeld R, Chen Q, Boone T, Simonet WS, et al. Reversal of cancer cachexia and muscle wasting by ActRIIB antagonism leads to prolonged survival. Cell. 2010;142(4):531-43.

22. Xu H, Crawford D, Hutchinson KR, Youtz DJ, Lucchesi PA, Velten M, McCarthy DO, Wold LE. Myocardial dysfunction in an animal model of cancer cachexia. Life Sci. 2011;88(9-10):406-10.

23. DeBoer MD, Zhu XX, Levasseur P, Meguid MM, Suzuki S, Inui A, Taylor JE, Halem HA, Dong JZ, Datta R, et al. Ghrelin treatment causes increased food intake and retention of lean body mass in a rat model of cancer cachexia. Endocrinology. 2007;148(6):3004-12.

24. Markison S, Foster AC, Chen C, Brookhart GB, Hesse A, Hoare SR, Fleck BA, Brown BT, Marks DL. The regulation of feeding and metabolic rate and the prevention of murine cancer cachexia with a small-molecule melanocortin-4 receptor antagonist. Endocrinology. 2005;146(6):2766-73.

25. Nazarian A, Cory E, Müller R, Snyder BD. Shortcomings of DXA to assess changes in bone tissue density and microstructure induced by metabolic bone diseases in rat models. Osteoporos Int. 2009;20(1):123-32.

26. Brandi ML. Microarchitecture, the key to bone quality. Rheumatology (Oxford). 2009;48(Suppl 4):iv3-8.

27. Marques EA, Mota J, Carvalho J. Exercise effects on bone mineral density in older adults: a meta-analysis of randomized controlled trials. Age (Dordr). 2012;34(6):1493-515.

28. Roth ES, Fetzer DT, Barron BJ, Joseph UA, Gayed IW, Wan DQ. Does colon cancer ever metastasize to bone first? a temporal analysis of colorectal cancer progression. BMC Cancer. 2009:9:274.

29. Morony S, Capparelli C, Sarosi I, Lacey DL, Dunstan CR, Kostenuik PJ. Osteoprotegerin inhibits osteolysis and decreases skeletal tumor burden in syngeneic and nude mouse models of experimental bone metastasis. Cancer Res. 2001;61(11):4432-6.

30. Coleman RE. Clinical features of metastatic bone disease and risk of skeletal morbidity. Clin Cancer Res. 2006;12(20 Pt 2):6243s-9s

31. Cormie P, Newton RU, Spry N, Joseph D, Taaffe DR, Galvão DA. Safety and efficacy of resistance exercise in prostate cancer patients with bone metastases. Prostate Cancer Prostatic Dis. 2013;16(4):328-35.

32. Zebaze R, Ghasem-Zadeh A, Mbala A, Seeman E. A new method of segmentation of compact-appearing, transitional and trabecular compartments and quantification of cortical porosity from high resolution peripheral quantitative computed tomographic images. Bone. 2013;54(1):8-20.

33. Zebaze RM, Libanati C, Austin M, Ghasem-Zadeh A, Hanley DA, Zanchetta JR, Thomas T, Boutroy S, Bogado CE, Bilezikian JP, et al. Differing effects of denosumab and alendronate on cortical and trabecular bone. Bone. 2014:59:173-9.

\section{Submit your next manuscript to BioMed Central and we will help you at every step:}

- We accept pre-submission inquiries

- Our selector tool helps you to find the most relevant journal

- We provide round the clock customer support

- Convenient online submission

- Thorough peer review

- Inclusion in PubMed and all major indexing services

- Maximum visibility for your research

Submit your manuscript at www.biomedcentral.com/submit 